\title{
A Cone of Inhomogeneous Second-Order Polynomials*
}

\author{
Robert Erdahl \\ Department of Mathematics and Statistics, Queen's University, \\ Kingston, Ontario K 7L 3N6, Canada \\ ErdahIR@QUCDN.QueensU.CA
}

Communicated by H. S. M. Coxeter

Abstract. Let $\mathscr{P}^{n}$ be the cone of quadratic functions

F1. $f=f_{0}+\sum f_{i} x_{i}+\sum f_{i j} x_{i} x_{j}, f_{i j}=f_{j i}$,

on $\mathbb{R}^{n}$ that satisfy the additional condition

$$
\text { F2. } f(z) \geq 0, z \in \mathbb{Z}^{n} \text {, }
$$

where $\mathbb{Z}$ denotes the integers. The coefficients and variables are assumed to be real and $1 \leq i, j \leq n$. The extent to which information on the convex structure of $\mathscr{P}^{n}$ can be used to determine the integer solutions of the equation $f=0$ for $f \in \mathscr{P}^{n}$ has been studied.

The root figure of $f \in \mathscr{P}^{n}$, denoted $R_{f}$, is the set of $n$-vectors $z \in \mathbb{Z}^{n}$ satisfying the equation $f(z)=0$. The root figures relate to the convex structure of $\mathscr{P}^{n}$ in an obvious way: if $R$ is a root figure, then

$$
F_{R}=\left\{q \in \mathscr{P}^{n} \mid R_{q}=R\right\}
$$

is a relatively open face with closure $\left\{q \in \mathscr{P}^{n} \mid q(r)=0, r \in R\right\}$. However, such formulas do not hold for all the relatively open and closed faces; this relates to some subtleties in the structure of $\mathscr{P}^{n}$.

Enumeration of the possible root figures is the central problem in the theory of $\mathscr{P}^{n}$. The group $G\left(\mathbb{Z}^{n}\right)$, of affine transformations on $\mathbb{R}^{n}$ leaving $\mathbb{Z}^{n}$ invariant, is the full symmetry group of $\mathscr{P}^{n}$. Classification of the root figures up to $G\left(\mathbb{Z}^{n}\right)$-equivalence

* This research was supported by the Natural Sciences and Engineering Research Council of Canada and the Advisory Research Committee of Queen's University. 
provides a complete solution to this problem, and this paper is concerned with some basic questions relating to such a classification.

The ideas in this study closely relate to the theory of $L$-polytopes in lattices as developed by Voronoi [V1], [V2], Delone [De1], [De2], and Ryshkov [RB]; $L$-polytopes, along with their circumscribing empty spheres (often referred to as holes in lattices), play a central role in the study of optimal lattice coverings of space. In addition, the theory of $\mathscr{P}^{n}$ makes contact with: (1) the theory of finite metric spaces, in particular hypermetric spaces [DGL1], [DGL2], and (2) a significant problem in quantum mechanical many-body theory related to the theory of reduced density matrices [E2]-[E4].

\section{Introduction}

Suppose that $E \subset \mathbb{R}^{n}$ is an ellipsoid. Then $E$ will be called empty if its interior contains no integer points. If, besides being empty, $E$ contains sufficiently many elements from $\mathbb{Z}^{n}$ so that these elements uniquely determine this quadratic surface, $E$ will be called perfect.

An empty ellipsoid determines an entire ray of $\mathscr{P}^{n}$, for let $f$ be some quadratic function such that the interior of $E$ is given by $\left\{x \in \mathbb{R}^{n} \mid f(x)<0\right\}$. Then $f$ has nonnegative values on $\mathbb{Z}^{n}$ and the ray generated by taking all positive scalar multiples of $f$ belongs to $\mathscr{P}^{n}$; this ray is uniquely determined by $E$. If $E$ is perfect, then the root figure $E \cap \mathbb{Z}^{n}$ must contain at least $N-1$ elements from $\mathbb{Z}^{n}$; $N=\left(\begin{array}{c}n+2 \\ 2\end{array}\right)$ is the dimension of the linear space of quadratic functions on $\mathbb{R}^{n}$. In the special case where $E$ is perfect, $f$ will be called a perfect element of $\mathscr{P}^{n}$.

More generally, an element $f \in \mathscr{P}^{n}$ will be called perfect if the subspace of quadratic functions given by

$$
Q\left(R_{f}\right)=\left\{q \text { quadratic } \mid q(r)=0, r \in R_{f}\right\}
$$

is one-dimensional. Elements which are not perfect will be referred to as imperfect.

The terms empty ellipsoid and perfect ellipsoid will often be used in the context of more general lattices $\Gamma \subset \mathbb{R}^{n}$, where $\operatorname{dim} \Gamma=1,2, \ldots$ These notions are generalized by substituting aff( $\Gamma$ ) (the affine hull of $\Gamma$ ) for $\mathbb{R}^{n}$ and $\Gamma$ for $\mathbb{Z}^{n}$ in the original definitions. For example, an empty ellipsoid in $\Gamma$ is an ellipsoid in aff $(\Gamma)$ containing no elements of $\Gamma$ in its interior. Other notions, such as perfect element, can be generalized in a similar way.

Proposition 1.1. $f \in \mathscr{P}^{n}$ is perfect if and only if $R_{f}$ is a maximal, proper root figure.

A proper root figure is neither empty nor equal to $\mathbb{Z}^{n}$. The root figures are partially ordered by containment and maximal refers to this ordering. It will be convenient to refer to maximal, proper root figures as perfect. Examples below show that perfect root figures can be either finite or infinite. 
Proof. Assume that $f$ is perfect. Then the linear space $S_{f}$ spanned by

$$
\left[1, r_{1}, \ldots, r_{n}, r_{1}^{2}, 2 r_{1} r_{2}, \ldots, r_{n}^{2}\right],\left[r_{1}, \ldots, r_{n}\right] \in R_{f}
$$

has codimension one in the $\left(\begin{array}{c}n+2 \\ 2\end{array}\right)$-dimensional space of coefficients. If $z \in \mathbb{Z}^{n}$ does not belong to $R_{f}$, then

$$
s_{z}=\left[1, z_{1}, \ldots, z_{n}, z_{1}^{2}, 2 z_{1} z_{2}, \ldots, z_{n}^{2}\right]
$$

does not belong to this linear space, and the only vector orthogonal to $S_{f}, s_{z}$ is the zero vector. That is, the only quadratic function $q$ satisfying the equations $q(z)=0$ and $q(r)=0, r \in R_{f}$, is the zero function with improper root figure $\mathbb{Z}^{n}$. Therefore $R_{f}$ is a maximal proper root figure.

That a maximal proper root figure is the root figure of a perfect element follows from Theorem 2.3 .

An element $f \in \mathscr{P}^{n}$ is said to be extreme if whenever $g, h \in \mathscr{P}^{n}$ are such that $f=g+h$, then both $g, h$ are nonnegative scalar multiples of $f$.

Proposition 1.2. The perfect elements of $\mathscr{P}^{n}$ are extreme.

Proof. Suppose that $f$ is perfect and $f=g+h$ with $g, h \in \mathscr{P}^{n}$. By property F2, $R_{f} \subset R_{g}, R_{h}$. Since $\operatorname{dim} Q\left(R_{f}\right)=1$, both $g$ and $h$ are scalar multiples of $f$. In fact, nonnegative scalar multiples since $g, h \in \mathscr{P}^{n}$. That is, $f$ is extreme.

There are perfect "ellipsoids" on the real line. An ellipsoid on $\mathbb{R}$ amounts to a pair of points; an empty ellipsoid to a pair of points with no integer points in between. All perfect ellipsoids on $\mathbb{R}$ consist of a pair of adjacent integer points $k$, $k+1$; this follows from the fact that the linear space of quadratic functions on $\mathbb{R}$ that are zero valued at $k, k+1$ is one-dimensional.

Suppose that $E$ is a perfect ellipsoid in $\mathbb{Z}^{n}$. Let $R=\mathbb{Z}^{n} \cap E$. In order that $Q(R)=\{q$ quadratic $\mid q(r)=0, r \in R\}$ be one-dimensional, $R$ must contain at least $\left(\begin{array}{c}n+2 \\ 2\end{array}\right)-1$ elements. However, the number of elements of $R$ cannot exceed $2^{n}$ by the following argument. The elements of $\mathbb{Z}^{n}$ can be grouped into $2^{n}$ equivalence classes depending upon the oddness or evenness of their $n$ components. Two distinct elements from the same equivalence class cannot belong to $R$ since their average is integer and interior to $E$, thus violating the condition that $E$ be empty. Since, for $n=2,3$,

$$
2^{n}<\left(\begin{array}{c}
n+2 \\
2
\end{array}\right)-1
$$

lattices having these dimensions cannot have perfect ellipsoids. 
In fact, there are no perfect ellipsoids in $\mathbb{R}^{n}$ when $2 \leq n \leq 5$ (Theorem 5.1 ). However, for $n=6,7$ there are examples of perfect ellipsoids (Theorem 5.3) which relate to the "deep holes" in the root lattices $E_{6}$ and $E_{7}$. The conditions for perfection are severe and perfect ellipsoids occur infrequently in lattices. It is this infrequent appearance of perfect ellipsoids that adds regularity, making $\mathscr{P}^{n}$ amenable to investigation.

Consider the function

$$
f(x)=\left(a_{1} x_{1}+\cdots+a_{n} x_{n}+k\right)\left(a_{1} x_{1}+\cdots+a_{n} x_{n}+k+1\right)
$$

where $a_{1}, a_{2}, \ldots, a_{n}, k \in \mathbb{Z}$ and $\operatorname{gcd}\left\{a_{1}, a_{2}, \ldots, a_{n}\right\}=1$. Since the coefficients are integer, the surface $f=0$ is a pair of parallel hyperplanes with no integer points lying in between. That is, this surface is a degenerate empty ellipsoid and $f \in \mathscr{P}^{n}$. Because of the arithmetic condition on the coefficients $a_{1}, a_{2}, \ldots, a_{n}$, the root figure consists of a pair of parallel sublattices of codimension one. It is an easy exercise to show that $R_{f}$ is a maximal proper root figure. By Proposition 1.1, $f$ is perfect. (In Section 2 the perfection of $f$ is established by a different argument involving the main Theorem 2.1.)

There are other classes of extreme elements besides the perfect elements. For example, by Theorem 2.1 the function

$$
f(x)=\left(\sqrt{2} x_{1}+x_{2}+\cdots+x_{n}+\sqrt{3}\right)^{2}
$$

is extreme. Since $1, \sqrt{2}, \sqrt{3}$ are linearly independent over the integers, the root figure $R_{f}$ is empty.

For each $f \in \mathscr{P}^{n}$, let $\varphi_{f}$ be the corresponding form. That is,

$$
\varphi_{f}=\sum f_{i j} x_{i} x_{j}
$$

Since $f$ satisfies condition $\mathrm{F} 2$, the form $\varphi_{f}$ satisfies the condition $\varphi_{f}(z) \geq 0$ for all $z \in \mathbb{Z}^{n}$.

Proposition 1.3. $\varphi_{f}$ is positive semidefinite.

Assume that $\varphi_{f}$ is positive definite and that $R_{f}$ is finite and nondegenerate. (A root figure $R$ is nondegenerate if the affine hull of $R$ is equal to $\mathbb{R}^{n}$.) Then $f$ has a unique minimum at, say, $c \in \mathbb{R}^{n}$, and by completing the square can be written

$$
f(x)=\varphi_{f}(x-c)-\left(\varphi_{f}(c)-f_{0}\right)
$$

Since $R_{f}$ was assumed nondegenerate $0<\varphi_{f}(c)-f_{0}=\rho^{2}$ and the surface $f=0$ is an empty ellipsoid in $\mathbb{R}^{n}$. However, if $\varphi_{f}$ is considered to be the metrical form 
for a lattice another interpretation is possible: the surface $f=0$ is an empty sphere of radius $\rho$ in a lattice $\Gamma_{f}$ determined by $\varphi_{f}$ (for an expanded discussion of empty spheres see [ER]). By changing the metric, the ellipsoid becomes a sphere and the vectors $z \in \mathbb{Z}^{n}$ become coordinate vectors for elements of a lattice $\Gamma_{f}$ with the basis defined as follows. Write the coefficient matrix for $\varphi_{f}$ as

$$
F=B^{\prime} B
$$

where $B$ is an $n \times n$ matrix; then take the columns of $B$ as the basis for $\Gamma_{f}$ (the basis is determined by $F$ only up to orthogonal equivalence). The matrix $F$ is the Gram matrix for the basis $B$, and $c$ is the coordinate vector for the center of the empty sphere referred to this basis.

At the 1924 International Congress of Mathematicians in Toronto, Delone gave an elegant definition for L-polytope [De1]: a polytope with vertices belonging to some lattice $\Gamma$ (and having the same dimension as $\Gamma$ ) is called an L-polytope in $\Gamma$ if it can be circumscribed by an empty sphere. Accordingly, $R_{f}$ gives the coordinates of the vertices of an L-polytope in the lattice $\Gamma_{f}$ with basis $B$. Voronoi introduced the notion of $L$-polytope in the second of his two famous memoirs "Nouvelles applications des paramètres continus à la théorie des formes quadratiques" [V1], [V2]; these two papers formed the basis for later work on the theory of optimal lattice coverings. In their book Sphere Packings, Lattices and Groups Conway and Sloane refer to the empty spheres circumscribing $L$-polytopes as holes in lattices [CS].

All of the $L$-polytopes in lattices of dimension $n=1,2, \ldots, 5$ can be studied uniformly and efficiently with dual systems of integer vectors. For $n \geq 6$ the family of $L$-polytopes that can be studied is large, but does not exhaust the entire set of $L$-polytopes. Dual systems were recently introduced in a series of three papers [RE3]-[RE5], the basic ideas and definitions being motivated by earlier work on the structure of $\mathscr{P}^{n}$ [E1]. Details on how $\mathscr{P}^{n}$ relates to the method of dual systems are given in Section 6. This discussion is in the context of the more general question of how root figures and therefore $\mathscr{P}^{n}$ relate to the theory of $L$-polytopes.

All of the $L$-polytopes in lattices of dimension $n \leq 4$ have been described earlier using other methods [ER], but these polytopes can be studied more efficiently using dual systems [RE5].

There are two other applications of $\mathscr{P}^{n}$ which are worth mentioning. The theory of finite metric spaces and $\mathscr{P}^{n}$ have recently been linked. In two interesting papers on hypermetrics and $L$-polytopes, Deza et al. establish a one-to-one correspondence between hypermetric spaces and $L$-polytopes [DGL1], [DGL2].

The study of $\mathscr{P}^{n}$ was originally taken up to shed light on a fundamental problem in quantum mechanical many-body theory. In his paper entitled "The structure of fermion density matrices," Coleman initiated the study of the mathematical properties of reduced density matrices [Col] (see also [Y]); the cone $\mathscr{P}^{n}$ was introduced to study some of the discrete geometric aspects of this theory [E1]. Additional details on how this physical theory relates to the cone $\mathscr{P}^{n}$ can be found in the literature [E2]-[E4] (see also [Da] and [DM]). 


\section{The Extreme Elements}

The main result on $\mathscr{P}^{n}$ is (a preliminary version can be found on p. 62 of [E1]):

Structure Theorem 2.1. An element $f \in \mathscr{P}^{n}$ is extreme if and only if it satisfies one of the three mutually exclusive conditions:

E1. $f$ is a positive constant.

E2. $f=\left(\alpha_{1} x_{1}+\alpha_{2} x_{2}+\cdots+\alpha_{n} x_{n}+\eta\right)^{2}$ where $\alpha=\left[\alpha_{1}, \ldots, \alpha_{n}\right]$ is not proportional to an integer vector.

E3. $f$ is perfect.

If $f$ is perfect its root figure is necessarily of the form

$$
R_{f}=R_{0}+\Gamma
$$

where

R1. $R_{0}$ is the collection of lattice points lying on a perfect ellipsoid in some lattice $\Gamma_{0} \subset \mathbb{Z}^{n} ; 1 \leq \operatorname{dim} \Gamma_{0} \leq n$.

R2. $\Gamma$ is a second sublattice of $\mathbb{Z}^{n}$ which together with $\Gamma_{0}$ satisfy the conditions $\mathbb{Z}^{n}=\Gamma_{0}+\Gamma,\left(\Gamma_{0}-\Gamma_{0}\right) \cap \Gamma=0$.

Moreover, any subset of $\mathbb{Z}^{n}$ of the form $R_{0}+\Gamma$, where $R_{0}, \Gamma$ satisfy conditions $\mathrm{R} 1$ and $\mathbf{R}_{2}$, is the root figure of a perfect element of $\mathscr{P}^{n}$.

The proof is left until Section 4. Condition $R 2$ ensures that elements $z \in \mathbb{Z}^{n}$ can be written uniquely as $z=\gamma_{0}+\gamma$ with $\gamma_{0} \in \Gamma_{0}, \gamma \in \Gamma$.

If $p$ is perfect, the surface $p=0$ is the cartesian product of a perfect ellipsoid in $\Gamma_{0}$ with the subspace $V=\operatorname{span} \Gamma$ having dimension $0,1,2, \ldots$; an ellipsoid, cylinder or generalized cylinder, depending on the dimension of $V$. The "axis" $V$ of this cylinder is also given by $\left\{x \in \mathbb{R}^{n} \mid \varphi_{p}(x)=0\right\}$, where $\varphi_{p}=\sum p_{i j} x_{i} x_{j}$ is the associated quadratic form. That $V$ has a basis consisting of integer vectors plays an important role in the following sections.

Consider again the function

$$
p(x)=\left(a_{1} x_{1}+\cdots+a_{n} x_{n}+k\right)\left(a_{1} x_{1}+\cdots+a_{n} x_{n}+k+1\right),
$$

where $a_{1}, a_{2}, \ldots, a_{n}, k \in \mathbb{Z}$ and $\operatorname{gcd}\left\{a_{1}, a_{2}, \ldots, a_{n}\right\}=1$. Choose some transverse line $L$ intersecting the parallel hyperplanes of the surface $p=0$ at, say, $z_{1}, z_{2} \in \mathbb{Z}^{n}$. Then

$$
R_{p}=R_{0}+\Gamma
$$

where $R_{0}=\left\{z_{1}, z_{2}\right\}$ and $\Gamma=\left[a_{1}, a_{2}, \ldots, a_{n}\right]^{1} \cap \mathbb{Z}^{n}$. However, $R_{0}$ is a pair of adjacent lattice points in the one-dimensional lattice $\Gamma_{0}=L \cap \mathbb{Z}^{n}$ and therefore a perfect "ellipsoid" in $\Gamma_{0}$ (see the discussion in Section 1 ), but $\Gamma_{0}, \Gamma$ clearly satisfy condition R2, so by Theorem $2.1 p$ is perfect.

The group $G\left(\mathbb{Z}^{n}\right)$ induces an action on $\mathscr{P}^{n}$ leaving it invariant, and the perfect 
elements of the form $\left(a_{1} x_{1}+\cdots+a_{n} x_{n}+k\right)\left(a_{1} x_{n}+\cdots+a_{n} x_{n}+k+1\right)$ all lie in a single orbit under this action. This is a special case of:

Corollary 2.2. For a given $n$, there is a one-to-one correspondence between the $G\left(\mathbb{Z}^{n}\right)$-orbits of perfect elements and the $G\left(\mathbb{Z}^{k}\right)$-equivalence classes of perfect ellipsoids in lattices of dimension $k=1,2, \ldots, n$.

There are several immediate consequence of Theorem 2.1.

Theorem 2.3. Any root figure $R \subset \mathbb{Z}^{n}$ can be represented as

$$
R=P_{1} \cap P_{2} \cap \cdots \cap P_{k}
$$

where $P_{i}$ is a perfect root figure.

Proof. It is easy to show that $\mathscr{P}^{n}$ has a base consisting of a compact convex set. Carathéodory's theorem [G, Theorem 1.7.1] can be applied and any element $f \in \mathscr{P}^{n}$ can be written as

$$
f=e_{1}+e_{2}+\cdots+e_{m}
$$

where $e_{i}$ is extreme and $m \leq n$. Assuming that it is not empty the root figure then has the form

$$
R_{f}=E_{1} \cap E_{2} \cap \cdots \cap E_{m}
$$

where $E_{i}$ is the root figure of $e_{i}$.

Suppose that $e_{1}, e_{2}, \ldots, e_{p}(p \leq m)$ are perfect. Let $g$ be the sum of the remaining extreme elements, which by Theorem 2.1 have the form $\left(\alpha_{1} x_{1}+\alpha_{2} x_{2}+\cdots+\right.$ $\left.\alpha_{n} x_{n}+\eta\right)^{2}$. When $g$ is zero the theorem obviously holds with $k=m=p$. When $g$ is nonzero the surtace $g=0$ is a proper affine subspace of $\mathbb{R}^{n}$, and $R_{g}$ is either a single point or a proper sublattice of $\mathbb{Z}^{n}$. The root figure $R_{g}$ can then be represented as the set of integer solutions of a system of linear equations, each of which has the form

$$
a_{1} x_{1}+a_{2} x_{2}+\cdots+a_{n} x_{n}+k=0
$$

where $a_{1}, a_{2}, \ldots, a_{n}, k \in \mathbb{Z}, \operatorname{gcd}\left\{a_{1}, a_{2}, \ldots, a_{n}\right\}=1$. Since

$$
\begin{aligned}
\left(a_{1} x_{1}+\cdots+a_{n} x_{n}+k\right)^{2}= & \frac{1}{2}\left(a_{1} x_{1}+\cdots+a_{n} x_{n}+k\right)\left(a_{1} x_{1}+\cdots+a_{n} x_{n}+k+1\right) \\
& +\frac{1}{2}\left(a_{1} x_{1}+\cdots+a_{n} x_{n}+k\right)\left(a_{1} x_{1}+\cdots+a_{n} x_{n}+k-1\right),
\end{aligned}
$$

$R_{g}$ can also be represented as the intersection of a finite number, say $q$, of perfect root figures. This finishes the proof since, then, $R_{f}$ can be written as the intersection of $k=p+q$ perfect root figures. 
The study of root figures quickly reduces to the study of nondegenerate root figures where $\operatorname{dim} R=n$. For suppose that $R \subset \mathbb{Z}^{n}$ is a root figure and $\operatorname{dim} R=k<n$. Write $\mathbb{Z}^{n}$ as $\mathbb{Z}^{k} \otimes \mathbb{Z}^{n-k}$ and let $\gamma \in G\left(\mathbb{Z}^{n}\right)$ be such that $\gamma(R) \subset \mathbb{Z}^{k} \otimes 0^{n-k}$. Then $\gamma(R)$ can be interpreted as a nondegenerate root figure in $\mathbb{Z}^{k}$. The statement of Theorem 2.3 is easily strengthened when $R$ is nondegenerate.

Representation Theorem 2.4. Suppose that $f \in \mathscr{P}^{n}$ has nondegenerate root figure $R_{f}$. Then $f$ can be represented as

$$
f=p_{1}+p_{2}+\cdots+p_{k},
$$

where $p_{i} \in \mathscr{P}^{n}$ is perfect and $k \leq n ; R_{f}$ has the corresponding representation

$$
R_{f}=P_{1}+P_{2}+\cdots+P_{k},
$$

where $P_{i}$ is the root figure of $p_{i}$.

Proof. The proof follows the same line as that for Theorem 2.3. The key point is that when $f$ is written as a sum of extreme elements, each of these must be perfect. Among the extreme elements it is only the perfect elements that have nondegenerate root figures. The bound on $k$ is a consequence of Caratheodory's theorem.

Corollary 2.5 [ER, Theorem 2.1]. An arbitrary nondegenerate root figure $R$ has the form

$$
R_{0}+\Gamma
$$

where

1. $R_{0}$ is the collection of lattice points lying on some empty ellipsoid in $\Gamma_{0} \subset \mathbb{Z}^{n}$; $1 \leq \operatorname{dim} R_{0}=\operatorname{dim} \Gamma_{0} \leq n$.

2. $\Gamma \subset \mathbb{Z}^{n}$ is a second sublattice of $\mathbb{Z}^{n}$ which together with $\Gamma_{0}$ form a direct sum decomposition of $\mathbb{Z}^{n} ; \mathbb{Z}^{n}=\Gamma_{0}+\Gamma,\left(\Gamma_{0}-\Gamma_{0}\right) \cap \Gamma=0$.

$R$ is finite if and only if $\operatorname{dim} \Gamma=0$.

Proof. By Theorem 2.4 an arbitrary nondegenerate root figure $R$ is the root figure of an element

$$
p=p_{1}+p_{2}+\cdots+p_{k},
$$

where $p_{i}$ is perfect. Let $\varphi_{p}$ be the form associated with $p$ and let $\varphi_{i}$ be the form associated with $p_{i}(i=1,2, \ldots, k)$. Only the case where $\varphi_{p}$ is indefinite need be considered since the result trivially holds when $\varphi_{p}$ is positive definite.

By Theorem 2.1 the surface $p_{i}=0$ is a cylinder or generalized cylinder with "axis" $V_{i}=\left\{x \in \mathbb{R}^{n} \mid \varphi_{i}(x)=0\right\}$, and $V_{i}$ has a basis consisting of integer vectors. The 
(nonzero) subspace

$$
V=\left\{x \in \mathbb{R}^{n} \mid \varphi_{p}(x)=0\right\}
$$

is equal to $V_{1} \cap V_{2} \cap \cdots \cap V_{k}$, so also has a basis consisting of integer vectors. Since $p$ must satisfy condition $\mathrm{F} 2$ it follows immediately that $p(x+v)=p(x)$ for $v \in \boldsymbol{V}$.

Let $\Gamma=\mathbb{Z}^{n} \cap V$ and introduce a second sublattice $\Gamma_{0}$ so that $\Gamma_{0}, \Gamma$ satisfy statement 2 of the theorem. The root figure $R$ can then be written as

$$
R=R_{0}+\Gamma
$$

where $R_{0}=\Gamma_{0} \cap R$.

Since $R$ was assumed to be nondegenerate, $\operatorname{dim} R_{0}=\operatorname{dim} \Gamma_{0}$. Since $V=$ span $\Gamma$, the surface $p=0$ restricted to aff $\Gamma_{0}$ is an empty ellipsoid $E$. Since $R_{0}=E \cap \Gamma_{0}, R_{0}$ is as in statement 1 of the theorem and is finite. It follows that $R$ is finite if and only if $\operatorname{dim} \Gamma=0$.

In the last step of the proof the following result was established.

Corollary 2.6. Suppose that $R_{f}$ is finite and nondegenerate. Then $\varphi_{f}$ is positive definite.

By Corollary 2.5 (and the discussion above) the study of root figures reduces to the study of finite nondegenerate root figures. By Corollary 2.6 and the comments in the introduction, this is equivalent to the problem of classifying $L$-polytopes in lattices.

Density Theorem 2.7. The perfect elements of $\mathscr{P}^{n}$ are dense in the extreme elements.

Proof. By Theorem 2.1 all of the extreme elements which are not perfect have the form

$$
\left(\alpha_{1} x_{1}+\alpha_{2} x_{2}+\cdots+\alpha_{n} x_{n}+\eta\right)^{2}
$$

These can be approximated with arbitrary accuracy by perfect elements of the form

$$
\alpha\left(a_{1} x_{1}+\cdots+a_{n} x_{n}+k\right)\left(a_{1} x_{1}+\cdots+a_{n} x_{n}+k+1\right)
$$

where $\alpha \in \mathbb{R},\left\{a_{1}, a_{2}, \ldots, a_{n}, k\right\} \subset \mathbb{Z}$, and $\operatorname{gcd}\left\{a_{1}, a_{2}, \ldots, a_{n}\right\}=1$.

\section{The Decomposition Lemma}

This section is about two linear subspaces of $\mathbb{R}^{n}$. Let

$$
V=\left\{x \in \mathbb{R}^{n} \mid \varphi_{f}(x)=0\right\}
$$


where $\varphi_{f}$ is the form associated with a typical element $f \in \mathscr{P}^{n}$. What turns out to be crucial is whether $V$ has a basis of integer vectors; if it does, $V$ and $\mathbb{Z}^{n} \cap V$ have a common basis. The second subspace is $V_{1}$, the minimal linear subspace containing $V$ that has a basis of integer vectors. The subspace $V_{1}$ is uniquely determined by $V$, and using orthogonal complements can be written as

$$
V_{1}=\left(\mathbb{Z}^{n} \cap V^{\perp}\right)^{\perp}
$$

Of course, if $V$ has a basis consisting of integer vectors (or is 0 ) $V_{1}=V$.

Decomposition Lemma 3.1. Let $f \in \mathscr{P}^{n}$. Then $f$ can be written as

$$
f=g+h
$$

where $g, h \in \mathscr{P}^{n}$ and have the following properties: $\left\{x \in \mathbb{R}^{n} \mid \varphi_{g}(x)=0\right\}=V_{1} ; h(x) \geq 0$ for all $x \in \mathbb{R}^{n}$.

Some preliminary results are needed for the proof.

Proposition 3.2. Let $f \in \mathscr{P}^{n}$. If $V \neq 0$, then $f$ is constant on the translates of $V$ by elements $x \in \mathbb{R}^{n}: f(x+v)=f(x), v \in V$.

Proof. Let $B$ be the closed ball of radius $\rho=\sqrt{n} / 2$ centered at the origin and let $M=\max _{x \in B} f(x)$.

For fixed $x \in \mathbb{R}^{n}$, the function $f(x+v)-f(x)$ is linear on $V$. Therefore, if the conclusion of the proposition does not hold there are elements $v \in V, x \in \mathbb{R}^{n}$ satisfying the condition

$$
M+f(x+v)-f(x)<0
$$

Assume that this is the case.

However, $\rho$ is the covering radius for $\mathbb{Z}^{n}$, so an element $z \in \mathbb{Z}^{n}$ can be chosen so that $z-v \in B$ and $f(z-v) \leq M$. The contradiction is achieved using the fact that $f$ must satisfy condition $\mathrm{F} 2$ :

$$
\begin{aligned}
0 \leq f(z) & =f(z-v+v)=f(z-v)+f(x+v)-f(x) \\
& \leq M+f(x+v)-f(x)<0
\end{aligned}
$$

(For the second equality the equation $\varphi_{f}(v)=0$ has been used.)

Proposition 3.3. Let $f \in \mathscr{P}^{n}$. Then

$$
M=\left\{m \in \mathbb{R}^{n} \mid f(m) \leq f(m+v), v \in V_{1}\right\}
$$

is an affine subspace of $\mathbb{R}^{n}$ satisfying the conditions $\mathbb{R}^{n}=M+V_{1}$ and $(M-M) \cap V_{1}=V$. 
Proof. Let $x \in \mathbb{R}^{n}, v \in V_{1}$. By Taylor's formula $f(m+t v)=f(m)+t(\nabla f(m))^{\prime} v+$ $t^{2} \varphi_{f}(v)$ and $m \in M$ if and only if $\nabla f(m) \perp V_{1}$. Being the solution set of a system of linear equations, $M$ is an affine subspace of $\mathbb{R}^{n}$.

For $x \in \mathbb{R}^{n}$ let $V_{1}(x)$ be the translate, $x+V_{1}$. By Proposition 3.2, the restriction of $f$ to $V_{1}(x)$ has a minimizing subset $M(x)$ so $V_{1}(x)=M(x)+V_{1}$. By the definition of $M, M(x) \subset M$ so $V_{1}(x) \subset M+V_{1}$. Since the union of all the translates $V_{1}(x)$ covers $\mathbb{R}^{n}, \mathbb{R}^{n}=M+V_{1}$.

By Proposition 3.2, $M(x)$ is a translate of $V$ so $V=M(x)-M(x) \subset M-M$. Since $V \subset V_{1}, V \subset(M-M) \cap V_{1}$. Now choose $v \in(M-M) \cap V_{1}$ arbitrarily. If $m \in M, t \in \mathbb{R}$, then $m+t v \in M$ and the following two inequalities hold:

$$
\begin{gathered}
f(m) \leq f(m+t v), \\
f(m+t v) \leq f(m+t v-t v)=f(m) .
\end{gathered}
$$

Then $f(m+t v)=f(m)$ for all real $t$ and $v \in V$. It follows that $(M-M) \cap V_{1}=V$, which completes the proof of the proposition.

Proposition 3.4. Let $f \in \mathscr{P}^{n}$. Then $f$ is nonnegative on the translates of $V_{1}$ by elements $z \in \mathbb{Z}^{n}: f(z+v) \geq 0, v \in V_{1}$.

To prove this statement the following result is needed.

Lemma. Suppose that $V$ is properly contained in $V_{1}$. Let

$$
K(\varepsilon)=\left\{v \in V_{1} \mid d(v, V) \leq \varepsilon\right\},
$$

an infinite, closed convex set in $V_{1}$. Then translates of $K(\varepsilon)$ by arbitrary elements $v \in V_{1}$ contain integer elements; $\{v+K(\varepsilon)\} \cap \mathbb{Z}^{n} \neq \varnothing$. Here $d(\cdot, \cdot)$ denotes the Euclidean distance.

Proof of the Lemma. Consider the lattice $\Gamma_{1}=\mathbb{Z}^{n} \cap V_{1}$. For $\beta>0$ the convex set $\beta K(\varepsilon) \subset V_{1}$ is symmetric about 0 and has infinite volume in $V_{1}$. Therefore, by Minkowski's celebrated Convex Body Theorem [GL, p. 40], the intersection $\Gamma_{1} \cap \beta K(\varepsilon)$ contains at least one nonzero element. The essential step of the proof is to show that $\operatorname{dim} \Gamma_{1} \cap \beta K(\varepsilon)=\operatorname{dim} V_{1}$. For if this is true, an arbitrary element $v \in V_{1}$ can be expanded in a basis, $\left\{u_{1}, u_{2}, \ldots, u_{k}\right\}$ for $V_{1}$, chosen from $\Gamma_{1} \cap \beta K(\varepsilon)$ :

$$
v=\xi_{1} u_{1}+\xi_{2} u_{2}+\cdots+\xi_{k} u_{k} .
$$

If integers $z_{1}, z_{2}, \ldots, z_{k}$ are chosen so that $\left|z_{i}-\xi_{i}\right| \leq \frac{1}{2}$, then and

$$
\gamma=z_{1} u_{1}+z_{2} u_{2}+\cdots+z_{k} u_{k} \in \Gamma_{1} \subset \mathbb{Z}^{n}
$$

$$
\gamma-v=\left(z_{1}-\xi_{1}\right) u_{1}+\left(z_{2}-\xi_{2}\right) u_{2}+\cdots+\left(z_{k}-\xi_{k}\right) u_{k} \in \frac{k}{2} \beta K(\varepsilon)
$$

With $\beta$ now set equal to $2 / k, \gamma \in v+K(\varepsilon)$ and $(v+K(\varepsilon)) \cap \mathbb{Z}^{n} \neq \varnothing$. 
Assume that $\operatorname{dim} \Gamma_{1} \cap \beta K(\varepsilon)<\operatorname{dim} V_{1}$. Then $V_{*}$, the subspace generated by the elements of $\Gamma_{1} \cap \beta K(\varepsilon) \subset \mathbb{Z}^{n}$, is properly contained in $V_{1}$. Since $V_{1}$ is the uniquely determined minimal subspace containing $V$ which is spanned by its integer elements, $V$ cannot be a subset of $V_{*}$. Choose $a \in V, a \notin V_{*}$ and consider the infinite cylinder $C(a, \delta)=\left\{x \in V_{1} \mid d(x, l(a)) \leq \delta\right\}$, where $l(a)$ is the line through $a$ and 0 . Assume that $\delta>0$ is chosen small enough so that $C(a, \delta)$ is contained in $\beta K(\varepsilon)$ but contains no elements of $V_{*} \cap \mathbb{Z}^{n}$. A second application of Minkowski's theorem shows that $C(a, \delta)$ must contain at least one nonzero element of $\Gamma_{1}$. Then $V_{*}$ does not contain $\Gamma_{1} \cap K(\varepsilon)$, and we have arrived at the contradiction. It follows that $\operatorname{dim} \Gamma_{1} \cap \beta K(\varepsilon)=\operatorname{dim} V_{1}$.

Proof of Proposition 3.4. If $V=V_{1}$, the result is easily established by applying Proposition 3.2. If $V$ is properly contained in $V_{1}$, the lemma is applied showing that $\{v+K(\varepsilon)\} \cap \mathbb{Z}^{n} \neq \varnothing$ and hence that $\{z+v+K(\varepsilon)\} \cap \mathbb{Z}^{n} \neq \varnothing$, when $z \in \mathbb{Z}^{n}$, $v \in V_{1}$. Choose a sequence $\varepsilon_{i}>0, i=1,2, \ldots$, converging to zero, and select $z_{i} \in z+v+K\left(\varepsilon_{i}\right)$. By Proposition 3.2 and the continuity of $f, \lim _{i \rightarrow \infty} f\left(z_{i}\right)=$ $f(z+v)$. Since $f\left(z_{i}\right) \geq 0$ it follows that $f(z+v) \geq 0$ and the proof is complete.

Proof of the Decomposition Lemma. If $V=V_{1}$ the Decomposition Lemma clearly holds: choose $g=f$ and $h=0$. The other possibility, that $V$ is properly contained in $V_{1}$, requires more work.

Because of the properties of $M$ quoted in Proposition 3.3, there are many ways to choose an affine subspace $N \subset M$ so that $N, V_{1}$ satisfy the conditions $\mathbb{R}^{n}=$ $N+V_{1}$ and $(N-N) \cap V_{1}=0$. Assuming such a choice has been made, elements $x \in \mathbb{R}^{n}$ can be written uniquely in the form

$$
x=n+v, \quad n \in N, \quad v \in V_{1},
$$

and functions $P_{N}, P_{V}$ on $\mathbb{R}^{n}$ can be defined so that $P_{N}(x)=n, P_{v}(x)=v$. It is easy to show that $P_{N}, P_{V}$ are affine.

Consider the quadratic functions defined by

$$
\begin{aligned}
& g(x)=f\left(P_{N}(x)\right) \\
& h(x)=\varphi_{J}\left(P_{V}(x)\right) .
\end{aligned}
$$

In the proof of Proposition 3.3 it was established that $m \in M$ if and only if $\nabla f(m) \perp V_{1}$. Since $N \subset M$,

$$
\begin{aligned}
f(n+v) & =f(n)+\nabla f(n)^{\prime} v+\varphi_{f}(v) \\
& =f(n)+\varphi_{f}(v) \\
& =g(x)+h(x) .
\end{aligned}
$$

Being positive on $\mathbb{R}^{n}, h \in \mathscr{P}^{n}$. That $g$ belongs to this cone can be seen as follows. Let $z \in \mathbb{Z}^{n}$ be chosen arbitrarily. Then $g(z)=f\left(P_{N}(z)\right)=f\left(z-P_{V}(z)\right) \geq 0$, the last inequality following from Proposition 3.4 . 
It is easy to see from the definition of $g$ that

$$
V_{1}=\left\{x \in \mathbb{R}^{n} \mid \varphi_{g}(x)=0\right\}
$$

This completes the proof of the Decomposition Lemma.

\section{Proof of the Structure Theorem}

Proceedings are initiated by noting that the Decomposition Lemma implies that an extreme element $f \in \mathscr{P}^{n}$ must satisfy at least one of the following two conditions:

C1. $f(x) \geq 0$ for $x \in \mathscr{P}^{n}$,

C2. $V=V_{1}$.

We first consider the extreme elements of $\mathscr{P}^{n}$ that satisfy condition C1. For this it is convenient to introduce the subcone $\mathscr{P}_{0}^{n} \subset \mathscr{P}^{n}$ of nonnegative quadratic functions on $\mathbb{R}^{n}$. The extreme elements of $\mathscr{P}_{0}^{n}$ are easily shown to be the positive constants and functions of the form

$$
\left(\alpha_{1} x_{1}+\alpha_{2} x_{2}+\cdots+\alpha_{n} x_{n}+\eta\right)^{2}
$$

where $\alpha=\left[\alpha_{1}, \alpha_{2}, \ldots, \alpha_{n}\right] \neq 0$. We must determine which from this list are extreme in $\mathscr{P}^{n}$.

E1. Suppose that $f$ is a positive constant; that is, $f$ satisfies condition E1 in the statement of Theorem 2.1. If $f=p+q$, with $p, q \in \not p^{n}$, then $\varphi_{p}=\varphi_{q}=0$ (see Proposition 1.3). By Proposition 3.2, p, q are nonnegative constant functions and therefore nonnegative scalar multiples of $f$. Therefore $f$ is extreme.

It is worth noting that the positive constants also satisfy $\mathrm{C} 2$. It will turn out that these are the only class of extreme elements satisfying both $\mathrm{C} 1$ and $\mathrm{C} 2$.

E2. Consider $f(x)=\left(\alpha_{1} x_{1}+\alpha_{2} x_{2}+\cdots+\alpha_{n} x_{n}+\eta\right)^{2}=(\langle\alpha, x\rangle+\eta)^{2}$, where $\alpha=\left[\alpha_{1}, \ldots, \alpha_{n}\right]$ is a nonzero integer vector with $\operatorname{gcd}\left\{\alpha_{1}, \ldots, \alpha_{n}\right\}=1$. Choose $k \in \mathbb{Z}$ so that $k-\frac{1}{2} \leq \eta \leq k+\frac{1}{2}$. Then a short computation shows that the real constants $a, b, c$, defined by the equality

$$
f(x)=a(\langle\alpha, x\rangle+k)(\langle\alpha, x\rangle+k-1)+b(\langle\alpha, x\rangle+k)(\langle\alpha, x\rangle+k+1)+c,
$$

are nonnegative. Therefore $f$ is not extreme. Since scaling by a positive number does not change this situation, $\left(\alpha_{1} x_{1}+\cdots+\alpha_{n} x_{n}+\eta\right)^{2}$ is not extreme if $\alpha$ is proportional to a nonzero integer vector.

Now consider the case where $\alpha$ is not proportional to an integer vector; that is, $f$ satisfies condition $\mathrm{E} 2$ in the statement of Theorem 2.1. Then $V_{1}=$ $\left(\mathbb{Z}^{n} \cap V^{\perp}\right)^{\perp}=\left(\mathbb{Z}^{n} \cap \alpha\right)^{\perp}=\mathbb{R}^{n}$. Assume that $f=p+q$ with $p, q \in \mathcal{P}^{n}$. Let $V_{p}, V_{q}$ be the subspaces where $\varphi_{p}, \varphi_{q}$ take the value zero. Since $\varphi_{f}=\varphi_{p}+\varphi_{q}, V \subset V_{p}, V_{q}$ and $\left(\mathbb{Z}^{n} \cap V_{p}^{\perp}\right)^{\perp}=\left(\mathbb{Z}^{n} \cap V^{\perp}\right)^{\perp}=\mathbb{R}^{n}$. An application of Proposition 3.4 shows that $p, q$ are nonnegative and therefore elements of $\mathscr{P}_{0}^{n}$. Since $f$ is extreme in $\mathscr{P}_{0}^{n}, p$ and $q$ 
are nonnegative scalar multiples of $f$. It follows that $f=\left(\alpha_{1} x_{1}+\cdots+\alpha_{n} x_{n}+\eta\right)^{2}$ is extreme in $y^{n}$.

E3. To complete the exhaustive description of extreme elements we consider the third mutually exclusive class. These are the extreme elements satisfying condition $\mathrm{C} 2$ but not $\mathrm{C} 1$, and turn out to be the perfect elements. The argument proceeds by establishing the truth of three separate statements which yield two alternative characterizations of the perfect elements.

A. If $f$ is extreme and satisfies $\mathrm{C} 2$ but not $\mathrm{C} 1$, then

$$
R_{f}=R_{0}+\Gamma
$$

where $R_{0}, \Gamma$ satisfy conditions $\mathrm{R} 1$ and $\mathrm{R} 2$ of the theorem.

Let $\Gamma=\mathbb{Z}^{n} \cap V$ and introduce a second sublattice $\Gamma_{0}$ so that $\mathbb{Z}^{n}=\Gamma_{0}+\Gamma$, $\left(\Gamma_{0}-\Gamma_{0}\right) \cap \Gamma=0$.

Let $E$ be the restriction of the surface $f=0$ to $V_{0}=\operatorname{aff}\left(\Gamma_{0}\right)$. Since $f$ satisfies condition $\mathrm{C} 2, V=\operatorname{span} \Gamma$, from which is drawn the following two conclusions. By Proposition 3.2 and the fact that $f$ does not satisfy $\mathrm{C}$, there is a region of $V_{0}$ where $f$ assumes negative values. Secondly, the surface $E$ bounds this region and is necessarily an ellipsoid. By condition $\mathrm{F} 2, E$ is an empty ellipsoid in $\Gamma_{0}$. By again using Proposition 3.2 it follows that

$$
R_{f}=R_{0}+\Gamma
$$

where $R_{0}=E \cap \Gamma_{0}$ is the collection of lattice points lying on the empty ellipsoid $E$ in $\Gamma_{0}$.

To complete the proof of statement $A$ it must be shown that $E$ is perfect in $\Gamma_{0}$. However, if $E$ were imperfect there would be a second quadratic surface $S \subset$ aff $\Gamma_{0}$, distinct from $E$, such that $R_{0} \subset \Gamma_{0} \cap S$. Let $g$ be a quadratic function on $\mathbb{R}^{n}$ with the property that the surface $g=0$ coincides with $S+V$. Then, with $\varepsilon>0$ sufficiently smaii, the two surfaces $f+\varepsilon g=0, f-\varepsilon g=0$ are generalized cylinders bounding regions of $\mathbb{R}^{n}$ void of integer elements. (These surfaces are slightly disturbed copies of the original surface $f=0$ which can be written as $E+V$.) Then $f+\varepsilon g, f-\varepsilon g \in \mathscr{P}^{n}$. Since

$$
f=\frac{1}{2}(f+\varepsilon g)+\frac{1}{2}(f-\varepsilon g)
$$

it follows that $f$ is not extreme. Since this contradicts one of our hypotheses, $E$ must be perfect.

B. If $R=R_{0}+\Gamma$ is a subset of $\mathbb{Z}^{n}$ such that $R_{0}, \Gamma$ satisfy conditions $\mathrm{R} 1$ and $\mathrm{R} 2$ of the theorem, then $R$ is the root figure of a perfect element of $\mathscr{P P}^{n}$.

Let $E$ be the perfect ellipsoid in $\Gamma_{0}$ containing $R_{0}$ and let $V=$ span $\Gamma$. Choose a nonzero quadratic function $f$ which has negative values only on the interior of 
the generalized cylinder $E+V$. Since this region is void of integer elements, $f \in \mathscr{P}^{n}$. For statement $\mathrm{B}$ to hold, $f$ must be perfect.

Let $q \in Q(R)$, the linear space of quadratic functions which are zero valued on $R$. For arbitrary $r \in R_{0}, \gamma \in \Gamma$, the function $q$ is zero valued on the line through $r$, $r+\gamma$. Since $E$ is perfect in $\Gamma_{0}, R_{0}$ has full dimension in $\Gamma_{0}$. Therefore, for arbitrary $x \in \operatorname{aff}\left(\Gamma_{0}\right), v \in V, q$ is constant valued on the line through $x, x+v$. Therefore the dimension of $Q(R)$ is equal to that of the linear space of quadratic functions on aff $\left(\Gamma_{0}\right)$ which are zero valued on $R_{0}$. Since by hypothesis this linear space is one-dimensional, $f$ is perfect.

C. The perfect elements of are are extreme and satisfy condition $\mathrm{C} 2$, but not $\mathrm{C} 1$.

By Proposition 1.2 the perfect elements of $\mathscr{P}^{n}$ are extreme. By the Decomposition Lemma we infer that they must satisfy one of the conditions $\mathrm{C} 1$ or $\mathrm{C} 2$. We have seen that the extreme elements satisfying $\mathrm{Cl}$ also satisfy either condition $\mathrm{E} 1$ or E2. However, these classes have degenerate root figures and are therefore imperfect. Thus perfect elements satisfy condition $\mathrm{C} 2$ but not $\mathrm{C} 1$.

Statements A, B, and C can be used to verify the assertions of the theorem regarding the perfect elements. This completes the proof of Theorem 2.1 .

Suppose $e$ is some element of a cone $C \subset \mathbb{R}^{n}$ with vertex at the origin. Then $e$ is said to be exposed if there is a supporting hyperplane $H$ such that $H \cap C$ contains only the ray generated by $e$. Exposed elements are necessarily extreme, but extreme elements need not be exposed. By working in the space of coefficients, additional information can be obtained on the extreme elements of $p^{n}$ : The extreme elements belonging to class 1 and 3 of Theorem 2.1 are exposed. The extreme elements belonging to class 2 are exposed if and only if the corresponding root figure is not empty. (Details are given in [E1].)

\section{Perfect Ellipsoids}

Theorem 5.1. There are no perfect ellipsoids in lattices of dimension 2, 3, 4, or 5 .

Since the proof is long and uses ideas considerably different than those developed here it will be published separately. (The result for the case $n=4$ is implicit in [ER]; see also Theorem 5 of [RE2].) As an immediate consequence, it follows that (see Theorem 2.1 and the discussion following):

Corollary 5.2. When $n \leq 5$ all perfect elements are of the form

$$
f(x)=\alpha\left(a_{1} x_{1}+\cdots+a_{n} x_{n}+k\right)\left(a_{1} x_{1}+\cdots+a_{n} x_{n}+k+1\right)
$$

where $\alpha \in \mathbb{R}$ is positive, $a_{1}, \ldots, a_{n}, k \in \mathbb{Z}$, and $\operatorname{gcd}\left\{a_{1}, \ldots, a_{n}\right\}=1$. 
The remainder of this section is a report on initial efforts to study perfect ellipsoids for the cases $n \geq 6$. The collection of integer points lying on a perfect ellipsoid is necessarily finite and nondegenerate, but by Corollary 2.6 , and the comments immediately following it, such root figures can be studied by examining $L$-polytopes in lattices. We have investigated the indecomposable root lattices, searching for $L$-polytopes contained in perfect holes, that is, the $L$-polytopes circumscribed by empty spheres which are perfect.

The deep holes in a lattice are those with maximum radius, all others are called shallow. It is the deep holes that are of greatest interest, the radii being equal to the covering radius of the lattice. (The center of a deep hole is a point at maximum distance from the lattice.)

Theorem 5.3. With the exception of the deep holes in $A_{1}, E_{6}, E_{7}$ all of the holes in the indecomposable root lattices $A_{n}(n \geq 1), D_{n}(n \geq 4), E_{6}, E_{7}, E_{8}$ are imperfect. The perfect holes in $E_{6}$ and $E_{7}$ contain L-polytopes with 27 and 56 vertices, respectively; these are the Gosset polytopes $2_{21}$ and $3_{21}$.

The notation $2_{21}, 3_{21}$ for these Gosset polytopes was introduced by Coxeter; they are described on pp. 202-204 of Regular Polytopes [Cox2] (see also pp. 21. 26 and 33 of $[\mathrm{Cox} 4]$ ).

In their most recent paper [DGL2], et al. report perfect ellipsoids in dimensions 15 and 16 related to the Barnes-Wall lattice and dimensions 22 and 23 related to the Leech lattice.

We will need a description of the holes in the indecomposable root lattices $A_{n}$ ( $n \geq 1), D_{n}(n \geq 4), E_{6}, E_{7}, E_{8}$. The development below is very close to that reported in [TD]; an alternate approach to obtain many of these results can be found in Chapter 21 of [CS]. For additional details on root lattices see Chapter 4 of $[\mathrm{CS}]$.

For any lattice $\Gamma$, the holes can be classified using the $L$-polytopes they contain, holes being considered different if they contain isometrically distinct species of $L$-polytopes. The collection of all $L$-polytopes having a vertex at the origin is called the star of $L$-polytopes at the origin. Each species of $L$-polytope is represented in this star, the number of representatives being a positive integral multiple of the number of vertices. This follows since each vertex of a given $L$-polytope can be translated to the origin yielding distinct representatives; the positive multiple exceeds one only when there are translationally inequivalent representatives of the same species.

The $L$-polytopes in the star at the origin fit together so that adjacent $L$ polytopes have a common facet. More generally, the collection of all $L$-polytopes form a tiling of space in which adjacent $L$-polytopes are glued together along common facets. These tilings are called $\boldsymbol{L}$-decompositions.

The centers of the empty spheres circumscribing the $L$-polytopes in the star have the following interesting characterization: they are the vertices of the Voronoi polytope $V$ of 0 . It is easy to show that this description of the Voronoi polytope is consistent with the usual one, that $V$ is the portion of space as close to the origin as to any other lattice point. Translating $V$ by $\gamma \in \Gamma$ produces the Voronoi polytope 
of the lattice point $\gamma$; the tiling formed by all the Voronoi polytopes is dual to the $L$-decomposition.

If an integral lattice $\Gamma$, containing the origin, is generated by its vectors of squared length two it is called a root lattice. These minimal vectors are called roots, and the set of all roots $\Phi$ is called a root system. By the Cauchy-Schwarz inequality, roots $\alpha, \beta$, when $\alpha \neq \pm \beta$, must satisfy the condition $\langle\alpha, \beta\rangle \in\{0, \pm 1\}$.

The root system $\Phi$ (and corresponding root lattice $\Gamma$ ) is decomposable if $\Phi$ can be written as an orthogonal direct sum, $\Phi=\Phi_{1} \oplus \Phi_{2}$, of root systems $\Phi_{1}, \Phi_{2}$. If $\Phi$ is not decomposable it is indecomposable.

We will need the following result on root lattices: if $\Gamma$ is a root lattice, then

$$
V=\left\{x \in \mathbb{R}^{n}|0 \leq| \alpha-\left.x\right|^{2}-|x|^{2}, \alpha \in \Phi\right\} .
$$

By replacing $\alpha \in \Phi$ by $\alpha \in \Gamma$ this converts to the usual formula for the Voronoi polytope at the origin for a general lattice, but involves an infinite system of inequalities. The simplification for root lattices follows almost directly from Coxeter's celebrated characterization of the fundamental region of an indecomposable reflection group as a simplex. (See the argument appearing on pp. $456-459$ of $[\mathrm{CS}]$.)

Notice that since $|\alpha-x|^{2}-|x|^{2}=|x|^{2}-2\langle x, \alpha\rangle=2(1-\langle x, \alpha\rangle), x \in V$ if and only if $\langle x, \alpha\rangle \leq 1$ for all $\alpha \in \Phi$. Therefore $V$ can be identified as the dual of the set of roots $\Phi$, and borrowing notation from convexity theory we write $V=\Phi^{0}$.

Let $\Gamma$ be a root lattice, and let $R$ be the vertex set of an $L$-polytope in the star at the origin. Denote by $\Gamma_{R}$ the lattice generated by the elements of $R$. Then $\Gamma_{R} \subseteq \Gamma$; if equality holds the $L$-polytope, or corresponding hole, will be called generating.

Proposition 5.4. The edge vectors of conv $R$ are roots, and $\Gamma_{R}$ is a root lattice. If $\Gamma$ is indecomposable, then so is $\Gamma_{R}$.

Proof. Let $x$ be the vertex of $V$ corresponding to the center of the empty sphere circumscribing $R$. Since $V=\Phi^{0}, x$ is also dual to the facet

$$
F=\{\alpha \in \Phi \mid\langle\alpha, x\rangle=1\}
$$

of $\Phi$. It is easy to see that conv $R$ intersects conv $\Phi$ in a pyramid with the apex at the origin and the base equal to conv $F$. This shows that the edges of conv $R$ which emanate from the origin, are precisely the roots $F$.

Additional copies of conv $R$ in the star are generated by translating successively the nonzero vertices of $R$ to the origin. Application of the above argument then shows that the edges emanating from any vertex, hence all edges, are roots. Since $\Gamma_{R}$ can be generated by the edges of conv $R$ it is necessarily a root lattice.

We now consider the case where $\Gamma$ is indecomposable. To establish that $\Gamma_{R}$ is also indecomposable we argue by contradiction, assuming that $\Gamma_{R}$ is decomposable and can be written $\Gamma_{R}=\Gamma_{1} \oplus \Gamma_{2}$. There is then a corresponding decomposition for the facet $F=R \cap \Phi: F=F_{1} \oplus F_{2}$. Since $\Gamma$ was assumed to be indecomposable 
there must be roots $\alpha \in F_{1}, \beta \in F_{2}, \gamma \in \Phi$ satisfying the conditions $\langle\alpha, \gamma\rangle$, $\langle\beta, \gamma\rangle= \pm 1$. By possibly replacing $\gamma$ by one of the roots $-\gamma, \alpha-\gamma, \beta-\gamma$, the signs of the scalar products can be adjusted so that $\langle\alpha, \gamma\rangle=\langle\beta, \gamma\rangle=1$. If $\delta$ is the root $\alpha+\beta-\gamma$ (the squared length of this vector is two), then

$$
\frac{1}{2}(\alpha+\beta)=\frac{1}{2}(\gamma+\delta)
$$

a formula showing that $\alpha, \beta$ do not generate an edge of conv $\Phi$. This is the contradiction that was sought: $\alpha, \beta$ do generate an edge of $F$. It follows that $\Gamma_{R}$ must be indecomposable.

The dual lattice $\Gamma^{*}$ of a lattice $\Gamma \subset \mathbb{R}^{n}$ is given by

$$
\Gamma^{*}=\left\{\lambda \in \mathbb{R}^{n} \mid\langle\lambda, \gamma\rangle \in \mathbb{Z}, \gamma \in \Gamma\right\}
$$

Notice that if $\Gamma$ is an integral lattice, for example a root lattice, then $\Gamma \subset \Gamma^{*}$.

Theorem 5.5. Assume that the $n$-dimensional root lattice $\Gamma \subset \mathbb{R}^{n}$ is indecomposable. Then $x \neq 0$ is a vertex of $V$ if and only if $x \in \Lambda^{*} \cap V$, where $0 \in \Lambda \subseteq \Gamma$ is some $n$-dimensional, indecomposable root lattice. If $R$ is the vertex set of an L-polytope with such a center $x \in \Lambda^{*} \cap V$, then $R$ generates $\Lambda$.

Using this theorem useful information can be obtained on the vertices of the Voronoi polytope of an indecomposable root lattice $\Gamma$. For example, suppose that $\Lambda \subseteq \Gamma(0 \in \Lambda)$ is an indecomposable root lattice of full dimension $n$. Since $\Gamma \subseteq \Lambda^{*}$, the elements of $\Lambda^{*}$ may be grouped into $\Gamma$-translation classes

$$
\text { [0], [1], [2], .., [d - 1], }
$$

the number of classes $d$ being equal to the index of $\Gamma$ in $\Lambda^{*}$. The translation class $[0]$ is equal to $\Gamma$ and is uninteresting. If $d \geq 2$ the sets $[1] \cap V, \ldots,[d-1] \cap V$ are nonempty and by Theorem 5.5 are $\Gamma$-translation classes of vertices of $V$; each corresponds to a distinct $\Gamma$-translation class of $L$-polytope. By considering all such lattices $\Lambda \subseteq \Gamma$, including $\Gamma$ itself, all of the $\Gamma$-translation classes of vertices and $L$-polytopes are generated.

If no such $n$-dimensional lattices $\Lambda$ are contained properly in $\Gamma$, then all of the holes of $\Gamma$ are generating (see Proposition 5.4). On the other hand, if $\Gamma$ is unimodular (and therefore equal to $E_{8}$ ) none of the holes in $\Gamma$ are generating.

Using some of the ideas in the following proof this last statement can be strengthened: if $\Gamma$ is integral, even and unimodular, then none of its holes are generating. A small but general deformation of such a lattice yields a general lattice in which all of the $L$-polytopes are simplicial. Since the resulting simplicial decomposition is a refinement of the $L$-decomposition for $\Gamma$, none of these $L$-simplexes are generating. In other words, this establishes the existence of a remarkable type of general lattice in which none of the $L$-simplexes are standard. The edge vectors of the individual $L$-simplexes generate sublattices of index 2 , 
$3, \ldots$ and are conveniently referred to as $L$-simplexes with double, triple, ... volume. (A simplex of volume $k$ has volume $k / n$ ! times that of a fundamental parallelopiped.)

Proof. Assume that $x$ is a vertex of $V$. If $R$ is the vertex set of the corresponding $L$-polytope, then $\gamma \in R$ must satisfy the equation

$$
|\gamma-x|^{2}-|x|^{2}=|\gamma|^{2}-2\langle\gamma, x\rangle=0
$$

Since $\Gamma$ is even it follows that $\langle\gamma, x\rangle \in \mathbb{Z}$, which in turn implies that $x \in \Gamma_{R}^{*}$. By Proposition $5.4 \Gamma_{R}$ is an indecomposable root lattice containing the origin. So if $\Lambda$ is taken equal to $\Gamma_{R}$ the only if side of the proof is settled.

Now assume that $x \neq 0$ belongs to $V \cap \Lambda^{*}$, where $\Lambda \subseteq \Gamma$ is an $n$-dimensional, indecomposable root lattice containing the origin. Notice that if $x \in \Phi_{\Lambda}\left(\Phi_{\Lambda}\right.$ is the corresponding root system), then $\langle x, \alpha\rangle \in\{0, \pm 1\}$. It will prove convenient to write

$$
\Phi_{\Lambda}=\Phi+\Phi_{0}+\Phi_{+},
$$

where $\Phi_{+}=\left\{\alpha \in \Phi_{\Lambda} \mid\langle x, \alpha\rangle=1\right\}$, etc. Clearly, $\Phi=-\Phi_{+} ;$it is argued below that $\Phi_{0}=\Phi_{+}-\Phi_{+}$. These formulas are significant because they establish that $\Lambda$ is generated by $\Phi_{+}$. Therefore $\Phi_{+}$has full dimension $n$ and $x$ is a vertex of $V$. In addition, it follows that the $L$-polytope centered at $x$ generates the lattice $\Lambda$.

Suppose that $\delta \in \Phi_{0}$ is not orthogonal to $\Phi_{+}$. Then there is a root $x \in \Phi_{+}$such that $|\langle\alpha, \delta\rangle|=1$. In this case $\beta=\alpha-\langle\alpha, \delta\rangle \delta$ is a second root belonging to $\Phi_{+}$ and the formula $\langle\alpha, \delta\rangle \delta=\alpha-\beta$ holds. Therefore $\langle\alpha, \delta\rangle \delta$, hence $\delta$ belong to the difference set $\Phi_{+}-\Phi_{+}$. Since $\Phi_{\Lambda}$ was assumed to be indecomposable, it then follows that $\Phi_{0} \cap \Phi_{+}^{\perp}$ is empty and $\Phi_{0}=\Phi_{+}-\Phi_{+}$.

Suppose that $R$ is the vertex set of an $L$-polytope in the star of $L$-polytopes at the origin for some general lattice $\Gamma$. The following serves as a useful test to determine whether the hole $R$ is imperfect.

Proposition 5.6. Assume that the dimension of $\Gamma$ exceeds one. If there is a vector $\delta \in \Gamma^{*}$ satisfying the condition $\langle\delta, \rho\rangle=0,1$ for all vertices $\rho \in R$, then $R$ is imperfect.

Proof. Since $\langle\delta, x\rangle$ is integer valued for $x \in \Gamma$, the quadratic function

$$
f(x)=\left(\delta_{1} x_{1}+\cdots+\delta_{n} x_{n}\right)\left(\delta_{1} x_{1}+\cdots+\delta_{n} x_{n}-1\right)
$$

is nonnegative on $\Gamma$, and by hypothesis zero valued on $R$. Suppose that $g$ is quadratic and such that the empty sphere circumscribing $R$ is given by the equation $g=0$. Then $f, g$ are linearly independent (the hypothesis $n \geq 2$ is used here) and zero valued on $R$. The dimension of $Q(R)=\{q$ quadratic $\mid q(x)=0\}$ is at least two and $R$ is imperfect.

It is now time to consider the details for the indecomposable root lattices. 
Lattice $A_{n}$. For $n \geq 1$

$$
A_{n}=\left\{x \in \mathbb{Z}^{n+1} \mid x_{1}+x_{2}+\cdots+x_{n+1}=0\right\}
$$

$n+1$ coordinates are used to describe these $n$-dimensional lattices [CS, pp. 108-117]. There are no $n$-dimensional, indecomposable root lattices $\Lambda$ properly contained in $A_{n}$. Therefore all the holes of $A_{n}$ are generating and the vertices of $V$ are elements of $A_{n}^{*}$. The lattice $A_{n}$ has index $n+1$ in $A_{n}^{*}$ and representatives of the various $A_{n}$-translation classes of $A_{n}^{*}$, chosen in $V$, are given by

$$
\begin{gathered}
{[0]=\left[0^{n+1}\right], \quad[1]=\frac{1}{n+1}\left[n,-1^{n}\right], \quad[2]=\frac{1}{n+1}\left[(n-1)^{2},-2^{n-1}\right], \ldots,} \\
{[n]=\frac{1}{n+1}\left[1^{n},-n\right] .}
\end{gathered}
$$

When $i \neq 0$ the general formula is given by

$$
[i]=\frac{1}{n+1}\left[j^{i},-i^{i}\right], \quad i+j=n+1
$$

the vertices of the corresponding $L$-polytope are generated by independently permuting the first $i$ and last $j$ coordinates of

$$
\left[1^{k}, 0^{i-k},-1^{k}, 0^{j-k}\right]
$$

where $1 \leq k \leq i, j$. Any other $L$-polytope is translationally equivalent to one of these.

As mentioned in the introduction, all of the holes in $A_{1}$, the one-dimensional lattice, are perfect. For $n \geq 2$ the vector

$$
\delta=\frac{1}{n+1}\left[n,-1^{n}\right]=[1,0, \ldots, 0]-\frac{1}{n+1}[1,1, \ldots, 1]
$$

belongs to the dual lattice $A_{n}^{*}$ (in fact, is a vertex of $V$ ). If $\rho$ is a vertex of any of the representative $L$-polytopes listed above, then $\langle\delta, \rho\rangle=0,1$, and by Proposition 5.6 all of the holes in $A_{n}, n \geq 2$, are imperfect.

Lattice $D_{n}$. For $n \geq 4$

$$
D_{n}=\left\{x \in \mathbb{Z}^{n} \mid x_{1}+x_{2}+\cdots+x_{n} \text { even }\right\}
$$

[CS, pp. 117-120], [RS]). There are no $n$-dimensional, indecomposable root lattices properly contained in $D_{n}$, so again all holes are generating and the vertices of $V$ are given by the formula $D_{n}^{*} \cap V$. The lattice $D_{n}$ has index 4 in $D_{n}^{*}$, and 
representatives in $V$ of the various translation classes of the elements of $D_{n}^{*}$ are given by

$$
\begin{array}{ll}
{[0]=\left[0^{n}\right],} & {[1]=\frac{1}{2}[1,1, \ldots, 1],} \\
{[2]=\frac{1}{2}[1,1, \ldots,-1],} & {[3]=\left[0^{n-1}, 1\right] .}
\end{array}
$$

The $L$-polytopes centered at [1], [2] are isometrically equivalent and correspond to deep holes. The vertices of the $L$-polytope centered at $[1]$ are all $(0,1)$-vectors whose entries have even sum; these $2^{n-1}$ vertices form the alternate vertices of an $n$-cube with edge length one. The other species of hole at [4] is shallow, the $L$-polytope being a cross polytope with $2 n$ vertices given by

$$
[4] \pm e_{i} \quad(i=1, \ldots, n)
$$

where $e_{i}=[0, \ldots, 0,1,0, \ldots, 0]$ is the unit vector along the $i$ th coordinate axis. The vector $\delta_{1}=\left[0^{n-1}, 1\right]$ belongs to $D_{n}^{*}$ and satisfies the condition $\left\langle\delta_{1}, \rho\right\rangle=0$, 1 when $\rho$ is a vertex of the $L$-polytope centered at [1]. Therefore this hole and all the deep holes of $D_{n}$ are imperfect. The vector $\delta_{3}=\frac{1}{2}[1,1, \ldots, 1]$ belongs to $D_{n}^{*}$ and satisfies the condition $\left\langle\delta_{3}, \rho\right\rangle=0,1$ when $\rho$ is a vertex of the $L$-polytope centered at [3]. Therefore the shallow holes, hence all the holes of $D_{n}$, are imperfect.

When $n=4$ the radii of the deep and shallow holes coincide, both equaling one. The alternate vertices of a 4-cube are also the vertices of a cross polytope, and all of the $L$-polytopes in $D_{4}$ are isometrically equivalent. The $L$-decomposition of $\mathbb{R}^{4}$ into copies of a single cross polytope is the regular honeycomb $\{3,3,4,3\}$ [Cox2, Sections 7.8 and 8.1$]$. The Voronoi polytope is the regular polytope $\{3,4,3\}$ with 24 vertices and 24 octahedral facets; the 24 -cell.

Lattice $E_{n}(n=6,7,8)$. In his paper entitled "Extreme forms," Coxeter replaces Euclidean $n$-space with Minkowskian $(n+1)$-space when describing the lattices $E_{n}, n=6,7,8[\operatorname{Cox} 1$, p. 419]. The Minkowski scalar product is given by

$$
\langle x, y\rangle=x_{1} y_{1}+x_{2} y_{2}+\cdots+x_{n} y_{n}-x_{n+1} y_{n+1},
$$

and $E_{n}$ can be realized by taking all the integer Minkowski vectors, $x$, satisfying the equation $\langle t, x\rangle=q$, where $q$ is an integer and $t$ is the time-like vector $[1,1, \ldots, 1 ; 3]$. It will prove convenient to use the copy of $E_{n}$ determined by the equation

$$
x_{1}+x_{2}+\cdots+x_{n}-3 x_{n+1}=-1 .
$$

The $n$ vectors obtained by permuting the first $n$ components of $\left[-10^{n-1} ; 0\right]$ belong to $E_{n}$ and form the vertices of a regular $(n-1)$-dimensional simplex $S_{n-1}$. This simplex forms the glue joint for a pair of neighboring $L$-polytopes in $E_{n}$. Table 1 describes the $L$-polytopes lying to the "right" and "left" of $S_{n-1} ; S_{n-1}$ is a facet of both. 
Table 1. Typical holes in $E_{n}$.

\begin{tabular}{|c|c|c|c|}
\hline Lattice & $E_{6}$ & $E_{7}$ & $E_{8}$ \\
\hline \multicolumn{4}{|c|}{ (the $L$-polytope to the left of $S_{n-1}$ ) } \\
\hline $\begin{array}{l}\text { Center } \\
\text { Radius }\end{array}$ & $\begin{array}{c}\frac{1}{3}\left[-2^{6} ; \sqrt{3}^{-3]}\right. \\
2 / \sqrt{3}\end{array}$ & $\frac{1}{4}\left[-1^{7} ;-1\right]$ & $\begin{array}{rr}\frac{1}{2}\left[-1^{*} ;\right. & -2] \\
i & \end{array}$ \\
\hline Vertices & 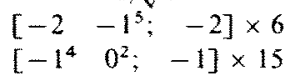 & {$\left[-1^{7} ; \stackrel{-2}{-}\right] \times 1$} & {$\left[\begin{array}{lll}-1^{7} & 0 ; & -2\end{array}\right] \times 8$} \\
\hline Description & 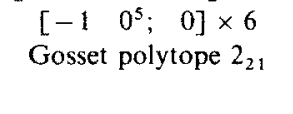 & $\begin{array}{c}{\left[\begin{array}{ccc}-1 & 0^{6} ; 0 & 0\end{array} \times 7\right.} \\
\text { Simplex with double } \\
\text { volume }\end{array}$ & $\begin{array}{c}{\left[\begin{array}{lll}-1 & 0^{7}: & 0\end{array}\right] \times 8} \\
\text { Cross polytope with } \\
\text { double volume }\end{array}$ \\
\hline \multicolumn{4}{|c|}{ (the $L$-polytope to the right of $S_{n-1}$ ) } \\
\hline $\begin{array}{l}\text { Center } \\
\text { Radius }\end{array}$ & $\begin{array}{c}\frac{1}{3}\left[1^{6} ; 3\right] \\
2 / \sqrt{3}\end{array}$ & $\frac{1}{2}\left[1^{7} ; 3\right]$ & $\frac{1}{3}\left[0^{8} ; 1\right]$ \\
\hline Vertices & 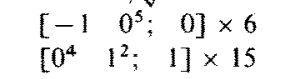 & 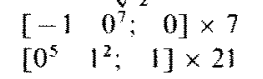 & {$\left[\begin{array}{lll}-1 & 0^{7} ; & 0\end{array}\right] \times 8$} \\
\hline & {$\left[\begin{array}{lll}0 & 1^{5} ; & 2\end{array}\right] \times 6$} & 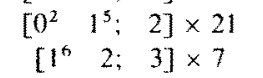 & {$\left[1^{8} ; 3\right] \times 1$} \\
\hline Description & Gosset polytope 221 & Gosset polytope $3_{21}$ & $\begin{array}{c}\text { Simplex with tripple } \\
\text { volume }\end{array}$ \\
\hline
\end{tabular}

The lattice $E_{6}$ is spectacular: all of the holes are equivalent, each containing a copy of the Gosset polytope $2_{21}$ with 27 vertices. In the $L$-decomposition these polytopes glue together along facets which are either regular simplexes (as illustrated in the table) or regular cross polytopes (which can easily be checked); this Gosset polytope has 72 simplicial facets and 27 facets which are cross polytopes [Cox2, pp. 202-204].

The lattice $E_{6}$ has index 3 in $E_{6}^{*}$. Since there is only one type of $L$-polytope, it follows from Theorem 5.5 that $2_{21}$ has its center in $E_{6}^{*}$ and is generating; more importantly, the vertices of the Voronoi polytope belong to $E_{6}^{*}$ and fall into two distinct $E_{6}$-translation classes. (It is also possible to deduce from this theorem that there are no proper six-dimensional sublattices in $E_{6}$ which are indecomposable root lattices.) Since $2_{2}$, has 27 vertices, each translation class contains 27 elements; the $L$-polytopes of these two classes are related by inversion. The Voronoi polytope is the reciprocal of the polytope $1_{22}$, described on p. 24 of [Cox4]. There are 54 $(=27 \times 2)$ Gosset polytopes in the star of $L$-polytopes at the origin.

There are two types of holes in $E_{7}$. The deep holes contain copies of the Gosset polytope $3_{21}$ with 56 vertices [Cox2, pp. 202-204]. Each Gosset polytope is glued to 126 equivalent Gosset polytopes along facets which are regular cross polytopes (that these six-dimensional cross polytopes serve as glue joints for a pair of Gosset polytopes is easily checked). In addition, each Gosset polytope is glued to 576 regular simplexes along simplicial facets (as shown in the table). These simplical $L$-polytopes are contained in the shallow holes of $E_{7}$.

As is apparent from the table, the $L$-simplex generates a sublattice of index two in $E_{7}$. Since the simplicial holes in $A_{n}$ are generating this sublattice can be identified 
with $A_{7}$ (see Theorem 5.5); these simplicial holes have minimal radius in $A_{7}$ and correspond to the translation classes $[1],[n]$ in the discussion of $A_{n}$. The total number of such $L$-simplexes in the star at the origin of $E_{7}$ is given by $\left|E_{7}\right| /\left|A_{6}\right|=576 ; A_{6}$ is the stability group of any one of these $L$-simplexes at the origin. Since each copy of $A_{7}$ accounts for $16=2 \times 8$ simplexes, there are 36 distinct ways in which $A_{7}$ appears as a sublattice containing the origin in $E_{7}$.

The lattice $E_{7}$ has index two in $E_{7}^{*}$. By Theorem 5.5 this accounts for a single translation class of generating $L$-polytope in the star of $L$-polytopes at the origin. This is the translation class of the Gosset polytope $3_{21}$, and it follows that there are 56 such polytopes in the star at the origin. Since there is only one translation class it can be inferred that $3_{21}$ has a center of symmetry. (This is also apparent from the representative of $3_{21}$ recorded in the table.)

The Voronoi polytope for $E_{7}$ has $632=56+576$ vertices and is the reciprocal of the polytope $2_{31}$ [Cox4, p. 27].

The $L$-polytope lying in a typical deep hole of $E_{8}$ is a regular cross polytope. Each of these cross polytopes is glued to 256 regular simplexes along each of its simplicial facets (as shown in the table). These $L$-simplexes are contained in the shallow holes, the only other type of hole in $E_{8}$.

Each cross polytope generates a copy of $D_{8}$ with index two in $E_{8}$. The star of $L$-polytopes at the origin contains $\left|E_{8}\right| /\left|D_{7}\right|=2160$ such cross polytopes; there are $135(=2160 / 16)$ distinct copies of $D_{8}$ containing the origin in $E_{8}$. Each simplex generates a copy of $A_{8}$ with index three in $E_{8}$. There are $\left|E_{8}\right| /\left|A_{7}\right|=17,280$ $L$-simplexes in the star of $L$-polytopes at the origin; there are $96(=1728 / 16)$ distinct copies of $A_{8}$ containing the origin in $E_{8}$. The Voronoi polytope is the reciprocal of the polytope $4_{21}$ [Cox2, p. 204], [Cox4, p. 36].

Since all of the holes in $E_{8}$ and the shallow holes in $E_{7}$ contain polytopes with an insufficient number of vertices $\left(<\left(\begin{array}{c}n+2 \\ 2\end{array}\right)-1\right)$, these holes are imperfect. (That these holes are imperfect also follows from the discussion of $A_{n}$ and $D_{n}$.) This leaves the deep holes in $E_{6}$ and $E_{7}$ as the only possible candidates for perfect holes.

Both types of hole in $E_{8}$ and the shallow hole in $E_{7}$ have been considerable upstaged in this discussion by the deep holes in $E_{6}$ and $E_{7}$, but these underdog holes are also distinguished; the edge vectors generate proper sublattices. As has been pointed out, the edge vectors of the simplex in $E_{7}$ generate a sublattice of index two (a simplex of double volume). The first instance of such an $L$-simplex is in five dimensions (see the discussion on p. 796 of Part I of [ER] and Sections 7 and 10 of $[R B]$ ), lattices in lower dimensions having only standard simplexes. There are examples showing that in dimension $2 k+1(k \geq 2)$ there are lattices with $L$-simplexes which generate sublattices of index $k$; when $k \geq 3$ it is not known whether this dimension is lowest possible for this phenomena. There are no examples of cross polytopes generating sublattices of index two in dimensions less that eight.

Completion of the proof of Theorem 5.3. We have just seen that, with the exception of the deep holes in $A_{1}, E_{6}$, and $E_{7}$, all of the holes in the irreducible 
root lattices are imperfect. Since there is only one type of hole in $A_{1}$ and it is perfect, all that remains is to verify the perfection of the deep holes in $E_{6}$ and $E_{7}$.

Notice that the restriction map establishes a linear isomorphism between the homogeneous quadratic forms on $\mathbb{R}^{n+1}$ and the quadratic functions on the plane

$$
x_{1}+x_{2}+\cdots+x_{n}-3 x_{n+1}=-1
$$

(which contains the copy of $E_{n}$ under investigation). Then, $R \subset E_{n}$ is the vertex set of a perfect $L$-polytope if and only if the linear space of forms on $\mathbb{R}^{n+1}$ which are zero valued on $R$ is one-dimensional. An equivalent characterization of perfection is that the matrices $r^{\prime} r, r \in R$ (with $r$ a row vector, $r^{\prime} r$ is an $(n+1) \times$ $(n+1)$ matrix), generate a linear space of codimension one in the space of real symmetric matrices. This last criterion is satisfied if the matrices $r^{\prime} r$, along with the metric matrix $M$ for the Minkowski metric, span this linear space.

It is convenient to denote the vertex sets for the Gosset polytopes $2_{21}, 3_{21}$ by

$$
\begin{array}{llrl}
a_{1}, a_{2}, \ldots, a_{n} & \text { with } & a_{1}=\left[\begin{array}{lll}
-1 & 0^{n-1} ; 0
\end{array}\right] \\
b_{12}, b_{13}, \ldots, b_{n-1 n} & \text { with } & b_{12}=\left[\begin{array}{llll}
1^{2} & 0^{n-2} ; 1
\end{array}\right] ; \\
c_{1}, c_{2}, \ldots, c_{6} & \text { with } & c_{1}=\left[\begin{array}{lll}
0 & 1^{5} ; 2
\end{array}\right] & \left(2_{21} \text { only }\right) ; \\
c_{12}, c_{13}, \ldots, c_{67} & \text { with } & c_{12}=\left[\begin{array}{llll}
0^{2} & 1^{5} ; 2
\end{array}\right] & \left(3_{21} \text { only }\right) ; \\
d_{1}, d_{2}, \ldots, d_{7} & \text { with } & d_{1}=\left[\begin{array}{llll}
2 & 1^{6} ; & 3
\end{array}\right] & \left(3_{21} \text { only }\right) .
\end{array}
$$

Let $E_{k m}, 1 \leq k \leq m \leq n+1$, be the standard basis for the real symmetric matrices:

$$
\begin{aligned}
& E_{k k}=\left\{\delta_{i k} \delta_{j k}\right\}, \\
& E_{k m}=\left\{\delta_{i k} \delta_{j m}+\delta_{i m} \delta_{j k}\right\}
\end{aligned}
$$

Let

$$
\begin{aligned}
& B=\sum_{k<m} b_{k m}^{\prime} b_{k m}, \\
& B_{1}=\sum_{1<k<m} b_{k m}^{\prime} b_{k m} \text {, } \\
& C= \begin{cases}\sum_{k} c_{k}^{\prime} c_{k} & \left(2_{21} \text { only }\right) \\
\sum_{k<m} c_{k m}^{\prime} c_{k m} & \left(3_{21} \text { only }\right),\end{cases} \\
& C_{1}= \begin{cases}c_{1}^{\prime} c_{1} & \left(2_{21} \text { only }\right) \\
\sum_{1<k} c_{1 k}^{\prime} c_{1 k} & \left(3_{21} \text { only }\right)\end{cases}
\end{aligned}
$$


Then the following formulas hold:

$$
\begin{aligned}
E_{11} & =a_{1}^{\prime} a_{1}, \\
E_{n+1, n+1} & =-M+\sum_{k} a_{k}^{\prime} a_{k}, \\
E_{1, n+1} & = \begin{cases}\frac{1}{10}\left[4 B-C-5 B_{1}+5 C_{1}-15 E_{11}-6 E_{77}\right] & \left(2_{21} \text { only }\right), \\
\frac{1}{30}\left[10 B-C-12 B_{1}-3 C_{1}-45 E_{11}-18 E_{88}\right] & \left(3_{21} \text { only }\right), \\
E_{12} & =b_{12}^{\prime} b_{12}-E_{11}-E_{22}-E_{n+1, n+1}-E_{1, n+1}-E_{2, n+1} .\end{cases}
\end{aligned}
$$

By permuting the indices $\{1,2, \ldots, n\}$ all of the basis elements are obtained. Thus the deep holes of $E_{6}$ and $E_{7}$ are perfect.

That the deep holes in $E_{6}$ are perfect has been known for a long time [E1]; the author is thankful to S. S. Ryshkov who was the first to notice that the deep holes in $E_{7}$ are also perfect.

\section{Holes in Lattices}

The problem of classifying root figures reduces to that of classifying the finite, nondegenerate root figures (see Corollary 2.5). Suppose that $R$ is such a root figure. Consider the relatively open face of $\mathscr{P}^{n}$ given by

$$
F_{R}=\left\{f \in P^{n} \mid R_{f}=R\right\}
$$

When $f \in F_{R}, \varphi_{f}$ is the metrical form for a lattice for which $R$ gives the coordinates of the vertices of an $L$-polytope (see Corollary 2.6 and the discussion in the introduction on $L$-polytopes). Define the domain of $R, \Phi_{R}$, to be the cone of all such metrical forms. The linear mapping $\Pi: f \rightarrow \varphi_{f}$ maps $F_{R}$ into $\Phi_{R}$. In fact, $\Pi$ establishes a one-to-one correspondence between the elements of $F_{R}$ and $\Phi_{R}$. Suppose that $\varphi \in \Phi_{R}$. If $c$ is the coordinate vector of the center of the $L$-polytope with vertices given by $R$, and $\rho$ is the radius, then

$$
f(x)=\varphi(x-c)-\rho^{2}
$$

belongs to $F_{r}$ and $\Pi$ is invertable.

Affine equivalence played a fundamental role in Voronoi's theory of $L$-types of lattices, the crowning achievement of his two mémoires [V2, Part IV]. He showed that the cone of positive definite forms can be partitioned into disjoint, relatively open convex subcones of varying dimension, called $L$-type domains, with the following properties:

A. On each of these subcones the affine structure of the $L$-decomposition of the corresponding lattices is constant. Subcones are arithmetically equivalent if and only if the affine structure of corresponding $L$-decompositions is identical. 
B. When the $L$-type domain has full dimension $N=\left(\begin{array}{c}n+1 \\ 2\end{array}\right)$ it is called general and the corresponding $L$-decomposition is simplicial. These domains are polyhedral. For each value of $n$ there are only a finite number of arithmetically inequivalent general $L$-type domains.

C. $L$-type domains of lesser dimension are called special and nonsimplicial $L$-polytopes appear in the corresponding $L$-decompositions. These are the faces of varying dimension of the general $L$-type domains, each being a face of two or more general domains.

The domain $\Phi_{R}$ is the union of all those $L$-type domains for which $R$ gives the coordinates of the vertex set of an $L$-polytope in the corresponding $L$-decomposition. In general, this union contains both general and special $L$-type domains.

Lemma 6.1. $\Phi_{R}$ is the union of a finite number of L-type domains.

Proof. By properties B and C the $L$-type domains of $\Phi_{R}$ are partitioned into a finite number of arithmetic classes. In turn, these classes can be partitioned into a finite number of $R$-equivalence classes which we now proceed to define.

Suppose that $\varphi_{1}, \varphi_{2} \in \Phi_{R}$. If $\Gamma_{1}, \Gamma_{2}$ are lattices determined by these forms, then $R$ gives the coordinates of the vertices of two $L$-polytopes, $P_{1} \in \Gamma_{1}, P_{2} \in \Gamma_{2}$. Define $\varphi_{1}$ to be $R$-equivalent to $\varphi_{2}$, if there is an affine transformation $T$, mapping $\Gamma_{1}$ onto $\Gamma_{2}$ preserving the structure of the $L$-decomposition, and such that $T\left(P_{1}\right)=$ $P_{2}$. By property $A$, the notion of $R$-equivalence extends to the $L$-type domains of $\Phi_{R}$, and is a refinement of arithmetic equivalence for these domains. For any given lattice, there are only a finite number of $L$-polytopes inequivalent under the group of lattice translations. It follows that the arithmetic classes of $L$-type domains of $\Phi_{R}$ partition into a finite number of $R$-equivalence classes.

More can be said about the $R$-equivalence classes of $\Phi_{R}$. Suppose that $\varphi_{1}, \varphi_{2}$ are $R$-equivalent and let $T$ be as above, mapping $P_{1}$ onto $P_{2}$. Then this transformation induces an affine transformation of coordinate vectors for the two lattices, $g_{T}$, leaving $R$ invariant. That is, $g_{T} \in G\left(\mathbb{Z}^{n}\right)$ and $g_{T}(R)=R$. Moreover, $g_{T}$ is equal to the identity transformation if and only if $\varphi_{1}, \varphi_{2}$ belong to the same $L$-type domain. The transformation $g_{T}$ belongs to the invariance group $G_{R} \subset G\left(\mathbb{Z}^{n}\right)$ of $R$ which must be finite, since by assumption $R$ is finite and nondegenerate. It follows that the $R$-equivalence classes of $L$-type domains in $\Phi_{R}$ are finite. Since there are only a finite number of $R$-inequivalent $L$-type domains in $\Phi_{R}$, there can only be a finite number of $L$-type domains in total.

Theorem 6.2. Assume that $R$ is a finite, nondegenerate root figure. Then the relatively open cones $F_{R}$ and $\Phi_{R}$ are polyhedral and linearly equivalent.

Proof. That $F_{R}$ and $\Phi_{R}$ are linearly isomorphic was argued above. That $\Phi_{R}$, hence $F_{R}$, is polyhedral follows from Lemma 6.1 and statements $\mathrm{B}$ and $\mathrm{C}$ above.

By Theorem 2.4 the extreme rays of $F_{R}$ are perfect. Since $F_{R}$ is open and polyhedral, an arbitrary element $f \in F_{R}$ can be written

$$
f=\sum_{P \supset \mathbf{R}} \lambda_{P} p
$$


where $\lambda_{P}>0$. The summation is over the perfect root figures containing $R$. That is, over the perfect elements belonging to $F_{R}(P$ is the root figure of the perfect element $p$ ). The corresponding formula for elements of $\Phi_{R}$ is given in:

Corollary 6.3. Assume that $R$ is a finite, nondegenerate root figure. Then an arbitrary form $\varphi \in \Phi_{R}$ can be written as

$$
\varphi=\sum_{P \supseteq R} \lambda_{P} \varphi_{P}
$$

where $\lambda_{P}>0$.

Suppose that $R \subset \mathbb{Z}^{n}$ is some collection of integer vectors (for the moment not a root figure). Define the dual of $R, R^{*}$ by

$$
R^{*}=\left\{r^{*} \in \mathbb{Z}^{n} \mid 0 \leq\left\langle r, r^{*}\right\rangle \leq 1, r \in R\right\}
$$

where $\left\langle r, r^{*}\right\rangle$ is the usual Euclidian scalar product. If $R$ satisfies the condition that

$$
R=\left(R^{*}\right)^{*},
$$

then $R, R^{*}$ is called a dual system of integer vectors. It is convenient to say that the dual system is nondegenerate if both $R$ and $R^{*}$ are nondegenerate [RE3][RE5].

If $R, R^{*}$ is a dual system, then both $R$ and $R^{*}$ contain the zero vector. If the system is nondegenerate, then both $R, R^{*}$ must be finite.

For each vector $r \in R$, let $\varphi_{r}$ be the form given by

$$
\varphi_{r}(x)=\left(r_{1} x_{1}+r_{2} x_{2}+\cdots+r_{n} x_{n}\right)^{2}
$$

$\varphi_{r}$ has unit rank.

A finite nondegenerate root figure containing the origin gives the coordinates of the vertices of an $L$-polytope in the star of $L$-polytopes at the origin.

Theorem 6.4. Assume that $n \leq 5$. Assume that $R$ is a finite, nondegenerate root figure containing 0 . Then $R$ is one of the partners of a nondegenerate dual system and the domain $\Phi_{R}$ is the collection of all forms

$$
\sum_{r \in R^{*}} \lambda_{r} \varphi_{r}
$$

where $\lambda_{r}>0$.

Proof. By Theorem 2.4 and Corollary 5.2, when $n \leq 5$, the extreme rays of $F_{R}$ are generated by the elements

$$
p(x)=\left(a_{1} x_{1}+\cdots+a_{n} x_{n}+k\right)\left(a_{1} x_{1}+\cdots+a_{n} x_{n}+k+1\right)
$$

satisfying the condition $p(r)=0, r \in R\left(a_{1}, a_{2}, \ldots, a_{n}, k \in \mathbb{Z}\right.$ and $\left.\operatorname{gcd}\left\{a_{1}, \ldots, a_{n}\right\}=1\right)$. 
Since $0 \in R, k$ must equal 0 or -1 . However, the first of these cases converts to the second by the replacement $a=\left[a_{1}, \ldots, a_{n}\right] \rightarrow-a$. Assuming that $k=-1$, $p(r)=0$ if and only if $0 \leq\langle a, r\rangle \leq 1$. Since this last condition must hold for all $r \in R$, it follows that $a \in R^{*}$. From this correspondence between the extreme rays of $F_{R}$ and the elements of $R^{*}$, it immediately follows that $\Phi_{R}$ is the collection of forms

$$
\sum_{r \in R^{*}} \lambda_{r} \varphi_{r}
$$

where $\lambda_{r}>0$.

The condition $r \in R$ if and only if $f(r)=0$, for $f \in F_{R}$, can now be restated as: $r \in R$ if and only if $0 \leq\langle a, r\rangle \leq 1$ for all $a \in R^{*}$. That is, $R=\left(R^{*}\right)^{*}$ and $R, R^{*}$ form a dual system.

Since, for $f \in F_{R}, \varphi_{f}$ is positive definite (Corollary 2.5), $R^{*}$ is nondegenerate. Since $R$ was assumed to be nondegenerate, the dual system $R, R^{*}$ is nondegenerate.

This theorem serves as the basis for a new method to enumerate $L$-polytopes in lattices. When the dimension $n$ satisfies the condition $n \leq 5$ all of the possible $L$-polytopes can be determined by first establishing a list of the nondegenerate dual systems up to arithmetic equivalence. (If $R, R^{*}$ is a dual system and $U \in G L(n, \mathbb{Z}), U^{*}=\left(U^{-1}\right)^{\prime}$, then $U R, U^{*} R^{*}$ is an arithmetically equivalent dual system.) Such a program has been carried out for $n \leq 4$ and the results are now available [RE4]. There are 16 arithmetically inequivalent, nondegenerate dual systems that can occur when $n=4$. Each partner gives the vertex set of an $L$-polytope, but there is one self-dual case where $R$ is arithmetically equivalent to $R^{*}$. The $31 \mathrm{~L}$-polytopes that result, are then grouped into $19 \mathrm{G}\left(\mathbb{Z}^{n}\right)$-equivalence classes, representing the various species of $L$-polytope that can occur in fourdimensional lattices.

Dual systems account for a large number of $L$-polytopes in higher-dimensional lattices. If $R, R^{*}$ is a nondegenerate dual system, then both $R$ and $R^{*}$ give the coordinates of the vertices of an $L$-polytope no matter what the dimension. However, the formula of Theorem 6.4 for $\Phi_{R}$ need not hold when $n \geq 6$. For all values of $n$, the $L$-polytopes in the root lattices $A_{n}, D_{n}$ can be represented as partners of dual systems; the simplexes of double and triple volume in $E_{7}$ and $E_{8}$ can also be represented as partners. The Gosset polytopes belong to the deep holes in $E_{6}$ and $E_{7}$ are obvious examples of $L$-polytopes that cannot be represented as partners (see Theorem 5.3). The cross polytope of double volume in $E_{8}$ is an example of an imperfect $L$-polytope which cannot be represented as a partner of a dual system (and will be reported on elsewhere).

\section{Acknowledgments}

The author is appreciative of the many helpful comments made by the reviewers. 


\section{References}

[Col] A. J. Coleman, The structure of fermion density matrices, Rev. Modern Phys. 35, 668-687 (1963).

[CS] J. H. Conway and N. J. A. Sloane, Sphere Packing, Lattices and Groups, Grundlehren der mathematischen Wissenschaften, Vol. 290, Springer-Verlag, New York, 1987.

[Cox1] H. S. M. Coxeter, Extreme forms, Canad. J. Math. 3, 391-441 (1951).

[Cox2] H. S. M. Coxeter, Regular Polytopes, 3rd edn., Dover, New York, 1973.

[Cox3] H. S. M. Coxeter, Regular and semi-regular polytopes, II, Math. Z. 188, 559-591 (1985).

[Cox4] H. S. M. Coxeter, Regular and semi-regular polytopes, III, Math. Z. 200, 3-45 (1988).

[Da] E. R. Davidson, Linear inequalities for density matrices, J. Math. Phys. 10, 725-734 (1969).

[DM] E. R. Davidson and W. B. McRae, Linear inequalities for density matrices, II, J. Math. Phys. 13, 1527-1537 (1972).

[De1] B. Delaunay [Delone], Sur la sphère vide, Proc. International Congress of Mathematicians, Toronto, 1924, University of Toronto Press, Toronto, pp. 695-700, 1928.

[De2] B. N. Delone, The geometry of positive quadratic forms, Uspekhi Mat. Nauk 3, 16-62 (1937); 4, $102-164$ (1938).

[DGL1] M. Deza, V. P. Grishukhin, and M. Laurent, Hypermetrics and L-polytopes, Research Report No. R.286, IASI-CNR, Roma Italy, 1990.

[DGL2] M. Deza, V. P. Grishukhin, and M. Laurent, Extreme hypermetrics and L-polytopes, Preprint (1991).

[E1] R. M. Erdahl, A convex set of second-order inhomogeneous polynomials with applications to quantum mechanical many body theory, Mathematical preprint \#1975-40, Queen's University, Kingston, Ontario.

[E2] R. M. Erdahl, Representability, Internat. J. Quant. Chem. 13, 697-718 (1978).

[E3] R. M. Erdahl, On the structure of the diagonal conditions, Internat. J. Quant. Chem. 13, 731-736 (1978).

[E4] R. M. Erdahl, Representability conditions, in Density Matrices and Density Functionals, D. Reidel, Pardrecht, 1987, pp. 51-75.

[ER] R. M. Erdahl and S. S. Ryshkov, The empty sphere, Canad. J. Math. 39, $794-824$ (1987); 40, 1058-1073 (1988).

[G] B. Grünbaum, Convex Polytopes, Interscience Series on Pure and Applied Mathematics, Vol. XVI, Wiley, New York, 1967.

[GL] P. M. Gruber and C. G. Lekkerkerker, Geometry of Numbers, North-Holland, Amsterdam, 1987.

[RB] S. S. Ryshkov and E. P. Baranovskii, The $C$-types of $n$-dimensional lattices and the five-dimensional primitive parallelohedrons (with applications to the theory of covering), Trudy Mat. Inst. Steklov 137, (1976); English transl. Proc. Steklov Inst. Math. 137, 1-140 (1978).

[RE1] S. S. Ryshkov and R. M. Erdahl, The geometry of the integer roots of some quadratic equations with many variables, Soviet Math. Dokl. 26, 668-670 (1982).

[RE2] S. S. Ryshkov and R. M. Erdahl, The laminar construction of $L$-polytopes in lattices, Uspekhi Mat. Nauk 44, 241-242 (1989).

[RE3] S. S. Ryshkov and R. M. Erdahl, Dual systems of integer vectors and their applications, Dokl. Akad. Nauk SSSR 314, 123-128 (1990).

[RE4] S. S. Ryshkov and R. M. Erdahl, Dual systems of integer vectors and their application to the theory of (0-1)-matrices, Trudy Mat. Inst. Steklov 196, 161-173 (1991).

[RE5] S. S. Ryshkov and R. M. Erdahl, Dual systems of integer vectors (general questions, applications to the geometry of positive quadratic forms), to appear in Mat. $S b$. (1991).

[RS] S. S. Ryshkov and S. S. Susbaev, The structure of the $L$-partition for the second perfect lattice, Mat. Sb. 116 (1981); English transl. Math. USSR-Sb. 44 (1983).

[TD] P. Terwiliger and M. Deza, Classification of finite connected hypermetric spaces, Graphs Combin. 3, 293-298 (1987).

[V1] G. F. Voronoi, Nouvelles applications des paramètres continus a là théorie des formes quadratiques. Premier mémoire, J. Reine Angew. Math. 133, 79-178 (1908). 
[V2] G. F. Voronoi, Nouvelles applications des paramètres continus a lá théorie des formes quadratiques. Deuxiéme mémoire, J. Reine Angew. Math. 134, 198-287 (1908); 136, 67-178 (1909).

[Y] C. N. Yang, The concept of off-diagonal long-range order and the quantum phases of liquid He and of superconductors, Rev. Modern Phys, 34, 694-704 (1962).

Received November 16, 1990, and in revised form August 5, 1991, and October 1,1991. 